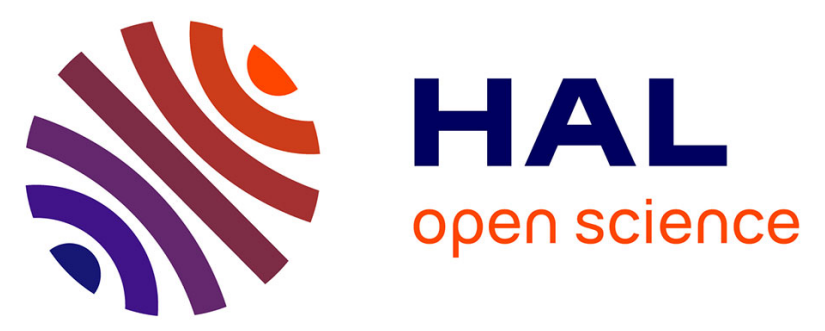

\title{
FlexiFingers: Multi-Finger Interaction in VR Combining Passive Haptics and Pseudo-Haptics
}

Merwan Achibet, Benoît Le Gouis, Maud Marchal, Pierre-Alexandre Léziart, Ferran Argelaguet Sanz, Adrien Girard, Anatole Lécuyer, Hiroyuki Kajimoto

\section{To cite this version:}

Merwan Achibet, Benoît Le Gouis, Maud Marchal, Pierre-Alexandre Léziart, Ferran Argelaguet Sanz, et al.. FlexiFingers: Multi-Finger Interaction in VR Combining Passive Haptics and Pseudo-Haptics. IEEE Symposium on 3D User Interfaces, Mar 2017, Los Angeles, United States. hal-01625154

\author{
HAL Id: hal-01625154 \\ https://hal.inria.fr/hal-01625154
}

Submitted on 27 Oct 2017

HAL is a multi-disciplinary open access archive for the deposit and dissemination of scientific research documents, whether they are published or not. The documents may come from teaching and research institutions in France or abroad, or from public or private research centers.
L'archive ouverte pluridisciplinaire HAL, est destinée au dépôt et à la diffusion de documents scientifiques de niveau recherche, publiés ou non, émanant des établissements d'enseignement et de recherche français ou étrangers, des laboratoires publics ou privés. 


\section{FlexiFingers: Multi-Finger Interaction in VR Combining Passive Haptics and Pseudo-Haptics}

\author{
Merwan Achibet \\ Inria/INSA Rennes, France \\ Ferran Argelaguet* \\ Inria Rennes, France
}

\author{
Benoît Le Gouis \\ IRISA/INSA Rennes, France \\ Adrien Girard \\ Inria Rennes, France
}

\author{
Maud Marchal* \\ IRISA/INSA Rennes, France \\ Anatole Lécuyer* \\ Inria Rennes, France
}

\author{
Pierre-Alexandre Leziart \\ ENS Rennes, France \\ Hiroyuki Kajimoto \\ The University of Tokyo, Japan
}

\begin{abstract}
3D interaction in virtual reality often requires to manipulate and feel virtual objects with our fingers. Although existing haptic interfaces can be used for this purpose (e.g. force-feedback exoskeleton gloves), they are still bulky and expensive. In this paper, we introduce a novel multi-finger device called "FlexiFingers" that constrains each digit individually and produces elastic forcefeedback. FlexiFingers leverages passive haptics in order to offer a lightweight, modular, and affordable alternative to active devices. Moreover, we combine Flexifingers with a pseudo-haptic approach that simulates different levels of stiffness when interacting with virtual objects. We illustrate how this combination of passive haptics and pseudo-haptics can benefit multi-finger interaction through several use cases related to music learning and medical training. Those examples suggest that our approach could find applications in various domains that require an accessible and portable way of providing haptic feedback to the fingers.
\end{abstract}

\section{INTRODUCTION}

Enabling users to grab and feel virtual objects with their own fingers would enhance interaction within virtual environments. However, haptic devices commonly used in virtual reality are unfit for precise dexterous manipulation and cannot provide such capabilities. As of today, the only systems capable of doing so are cumbersome and costly multi-finger devices such as active exoskeletons.

Simpler passive interfaces based on minimalist props can be used to stimulate the user's sense of touch during 3D interaction and offer a low-cost and lightweight alternative to active feedback $[7,23,15,2]$. While such approaches incorporate haptic feedback in an accessible manner, they are either limited to coarse grasping since fingers are not distinguished or they are grounded devices that limit the user's mobility. In this paper, we explore how passive haptics can contribute to multi-finger interaction with a novel lightweight elastic interface, the FlexiFingers, that handles the digits individually (see Figure 1). In this way, users can perform precise multi-finger tasks and receive variable forces on each digit.

Our contributions can be summarized as follows: (1) a novel elastic exoskeleton that enables multi-finger interaction and renders individual forces to several digits in a low-cost and portable manner by leveraging passive haptics, (2) an accompanying pseudo-haptic approach that enriches this passive feedback and simulates various levels of stiffness when manipulating deformable objects.

In this paper, we first present an overview of previous work related to multi-finger haptics. Then, we describe our novel device as well as the associated pseudo-haptic approach. Finally, we present several use cases that illustrate how such a device can contribute to richer interaction scenarios.

*e-mail:\{maud.marchal,fernando.argelaguet_sanz,anatole.lecuyer\}@inria.fr

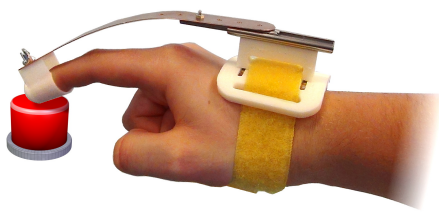

(a)

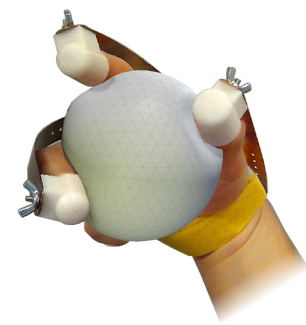

(b)
Figure 1: FlexiFingers, our passive multi-finger haptic device. (a) Single-finger configuration for simulating basic tasks such as pushing a button. (b) Three-finger configuration for simulating more complex tasks such as clay sculpting with several digits.

\section{Related Work}

Active multi-finger haptic devices - Common desktop haptic devices are generally unable to provide multi-finger force feedback. At most, they may be equipped with a thimble for conducting single-finger tasks [25], custom gripper handles to simulate basic grasping [4] or combine multiple devices to pinch objects [20]. Other desktop interfaces have been specifically designed for dexterous interaction, such as MasterFinger [18], DigiHaptic [9] and Spidar [28]. Those grounded systems may however restrain the users' mobility. Conversely, haptic exoskeletons $[8,19]$ directly mounted on the users' bodies allow them to move freely in larger immersive spaces but they are often characterized by complex mechanical designs. There are only a few commercial multi-finger exoskeleton available at the moment ${ }^{1,2}$. In our work, we explore how a similar feedback to multi-finger exoskeleton can be provided in a compact and affordable way by leveraging passive elastic components.

Passive haptics as an alternative - Passive haptics engages users haptically without relying on costly active devices. Instead, it leverages the material properties of inert props, suc as a proxy sponge to simulate sculpting [26]. For object manipulation, Paljic et al. [21] developed a passive Spidar with a string-mounted brake that generates friction when gripped. Similarly, the Virtual Mitten is an interaction metaphor making use of a spring-loaded hand-exerciser to simulate grasping forces [2]. However, these devices focus on coarse grasping and do not deliver haptic feedback to each finger independently. Other passive devices do distinguish the digits: Pihuit et al. [23] used a foam ball with pressure sensors under each fingertip to control a virtual hand and the HandNavigator [15] is a modified 3D mouse with elastic finger pedals. To provide users with a larger workspace, Koyama et al. [14] developed a partially passive exoskeleton that links fingertips to an elastic torsional shaft on the back of the hand. However, they use active clutches to hold

\footnotetext{
${ }^{1}$ CyberGlove Systems (www. cyberglovesystems. com)

${ }^{2}$ Dexta Robotics (www. dextarobotics.com/)
} 
up the fingers when necessary. Conversely, the device that we propose is only composed of passive components in order to minimize its complexity and bulkiness, and it leverages pseudo-haptics in order to alleviate the limitations of passive feedback.

Pseudo-haptics for enriching passive feedback - Passive haptics lacks flexibility since the material of the props cannot be changed on the fly to simulate various virtual properties. Therefore, methods based on the dominance of vision over proprioception have been proposed to alter the perceived response. Most notably, pseudohaptics is an approach that induces haptic cues by mismatching the real motion of the user and its visual restitution within the virtual environment [16]. For example, the Elastic-Arm [1] is a rubber armature that pulls the users' hands towards their body and plays on visual feedback to simulate different levels of stiffness when pushing virtual surfaces.

Recent research efforts also focused on the application of pseudo-haptics to dexterous interaction. Ban [3] simulated different shapes by warping the virtual representation of a real prop as well as the position of the users' finger while they follow its outline. For object grasping, Kimura et al. [13] conducted preliminary research in 2D by modulating visual feedback with respect to the pressure applied on a passive prop and the Virtual Mitten [2] leverages pseudo-haptics in 3D by altering visual feedback so that users apply different levels of effort on a passive input device. In those cases however, fingers are not distinguished. In this paper, we aim at bringing pseudo-haptic feedback to the separate digits in order to support scenarios requiring multi-finger interaction.

\section{A NOVEL PASSIVE MULTI-FINGER INTERFACE}

We propose the FlexiFingers, a novel passive hand-worn device that delivers haptic feedback to individual digits (see Figures 1 and 2). Associated to this device, we use pseudo-haptics to simulate different levels of stiffness when manipulating virtual objects. This approach follows up on previous work [2] that simulated coarse grasping and extends this principle to support multi-finger interaction.

The FlexiFingers device (see Figure 2) is composed of independent finger modules made of a bendable metal strip ending with a thimble. The metal strips offer a range of motion for the fingertip of $7.3 \mathrm{~cm}$. The full range can be reached with a force of $2.5 \mathrm{~N}$. A variable number of those modules is plugged on a base plate attached to the back of the hand with a velcro band. A smaller separate plate is on the thumb. Each module is anchored to the plate on a rail slider so that it can accompany curling motions without blocking the finger (Figure $2 b$ ). The top part of the thimbles also comprises a slot in which vibration motors could be inserted to simulate additional haptic effects at low cost.

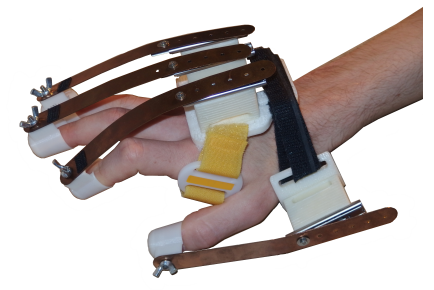

(a)

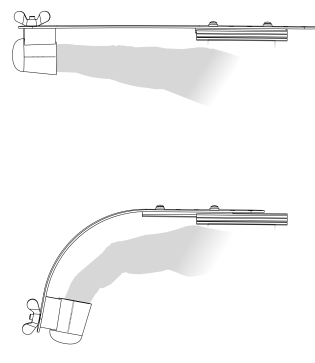

(b)
Figure 2: Our passive multi-finger device. (a) Bendable strips provide force feedback when the fingers curl. (b) Each module is mounted on a rail slider to accompany the digit during those curling motions.
The finger modules generate passive haptic feedback by constraining the digits towards a flat rest position. In consequence, fingers undergo an opposing elastic force that we can exploit for simulating haptic effects related to object manipulation such as grip force or stiffness. Due to the elastic nature of this device, the intensity of the feedback depends on the degree of curling of each finger. The pseudo-haptic effect described in a later section exploits this fact to induce different sensations depending on the properties of the virtual object that the user is interacting with. The FlexiFingers device aims at circumventing the drawbacks of existing multi-finger haptic interfaces and the following requirements motivated its design.

Size and weight - A wearable haptic interface should be compact and lightweight enough so as not to get in the way of the tasks being carried out. Here, the lack of motorization of passive haptics significantly reduces the weight of the device: a three-finger configuration only weights 100 grams. Concerning size, the bulk of the system occupies a vertical space of 4 centimeters on the back of the hand. The thin bendable strips (with dimensions $15 \mathrm{~cm} \times 1.5 \mathrm{~cm}$ $\times 0.5 \mathrm{~mm}$ ) run close to the finger so as not to hamper users with a bulky support armature.

Cost and complexity of the design - The device should remain affordable compared to commercial haptic systems as well as easy to produce. Our prototype is composed of simple parts which total price is under $\$ 30$. Its key components, the deformable strips that provide haptic feedback, are made of phosphor bronze, a common and affordable alloy. The base plates and thimbles are 3D-printed which makes them cheap to produce as well as easy to adapt to different morphologies.

Feedback quality - The haptic feedback delivered by the device should be consistent with object grasping. Different iterations led to the current prototypes. The first version relied on rubber bands pulling the fingertips (Figure 3a). In a second prototype, the rubber bands were raised to provide forces consistent with efforts expected from grasping, ie. normal to the fingertips (Figure 3b), and a third prototype separated the fingers for more mobility (Figure 3c). Contrarily to those first versions, the current prototype uses deformable strips that oppose curling motions in a more compact manner.

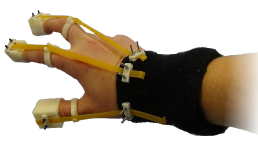

(a)

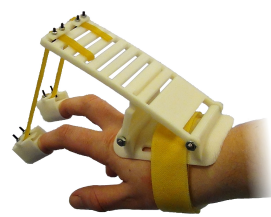

(b)

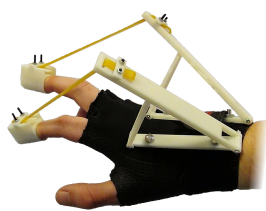

(c)
Figure 3: Different iterations of the FlexiFingers. (a) First prototype with rubber bands pulling the fingers. (b) Second prototype raising the anchor points of the rubber bands to simulate grasping forces more truthfully. (c) Third prototype with separated fingers for more comfort.

Modularity - The device should accommodate different numbers of fingers depending on the context. Indeed, pushing a virtual button may only require a single finger (Figure 1a) while sophisticated precision grasps may require three fingers or more (Figure 1b). Here, various numbers of finger modules can be plugged on the base plate depending on the interaction scenario. The intensity of the haptic feedback can also be tuned either by choosing bendable strips with an appropriate stiffness or by stacking several strips together. In addition, we explored pseudo-haptics to vary the perceived haptic feedback, as detailed in the following section. 


\section{VARYING THE PERCEIVED EFFORT USING PSEUDO- HAPTICS}

The FlexiFingers leverages passive haptics to render forces to the digits, yet the stiffness of its bending strips is constant. Thus, we rely on pseudo-haptics to render different levels of stiffness and simulate a wider range of virtual objects. We first present the coupling between users and their virtual hand, then we describe the visual mismatch that makes our effect, and finally we expand on how to author pseudo-haptics-enabled objects.

Virtual coupling - A 3D virtual hand model embodies the user's hand. We describe each virtual finger $n \in\{1,2,3,4,5\}$ with a virtual flexion parameter $v_{n} \in[0,1]$ such that $v_{n}=0$ corresponds to an extended finger and $v_{n}=1$ corresponds to a curled finger. Those parameters are reflected on the $3 \mathrm{D}$ model according to a curling rule similar to Rijpkema's [24]: the proximal joint of finger $n$ rotates with respect to $v_{n}$ within predefined bounds and following joints are rotated by $2 / 3$ of their parent joint's rotation.

A LeapMotion sensor ${ }^{3}$ tracks the position of the user's wrist and the orientation of the finger joints. The wrist data is mapped as is in the virtual environment. For finger curling, we extract real flexion parameters $r_{n} \in[0,1]$ corresponding to the actual flexion of the user's fingers. As it stands, a truthful 1:1 coupling between real and virtual hands can thus be simply formulated as:

$$
\forall n, \quad v_{n}=r_{n}
$$

Stiffness rendering - Pseudo-haptics consists in altering the visual feedback resulting from the user's actions in order to express haptic properties [10]. Here, we consider stiffness so that users can distinguish soft objects from firm ones.

We induce this sensation by altering the Control/Display ratio of the virtual fingers while users manipulate an object. Increasing the ratio slows down fingers, which suggests a stiffer object. Users need to compensate their own posture to reach the same amount of visual deformation for various objects. Thus, a firmer object requires to amplify one's curling and, in consequence, the FlexiFingers generates stronger haptic cues. We call $s_{n}$ the ratio that scales the flexion of virtual finger $n$, such that:

$$
\forall n, \quad v_{n}=r_{n} \cdot s_{n}
$$

A potential issue with this mapping between real and virtual hands arises from the fact that our passive device provides force feedback as soon as the fingers curl. In consequence, users already perceive an effort while closing the hand around a virtual object even though they are not touching its surface yet. This unrealistic state can be skipped by automatically curling the virtual fingers around objects when they are at reach. In this way, the neutral flat posture of the user's real hand is mapped to the grasping posture of the virtual hand. The flexion parameter $c_{n}$ corresponding to finger $n$ just touching the surface of the object is obtained by casting rays along the trajectory of each finger. The finger then curls within the remaining flexion span $1-c_{n}$ such that:

$$
\forall n, \quad v_{n}=c_{n}+\left(1-c_{n}\right) \cdot r_{n} \cdot s_{n}
$$

In any case, the pseudo-haptic effect plays on a mismatch between real and virtual hands, which might seem counter-intuitive. However, previous work did not report any negative effect from users using a similar approach to simulate grasping [2]. Contextspecific cases for which the real/virtual offset might be more noticeable could be evaluated, such as with very soft or rigid objects.

Pseudo-haptic authoring - Rendering the stiffness of an object with this pseudo-haptic approach requires to bind it to appropriate haptic parameters. We embed the haptic data governing this effect into the mesh of the virtual object. In this way, a stiffness value can be obtained for any point on its surface by interpolation, as usually

${ }^{3}$ Leap Motion Inc. (www. leapmotion.com)

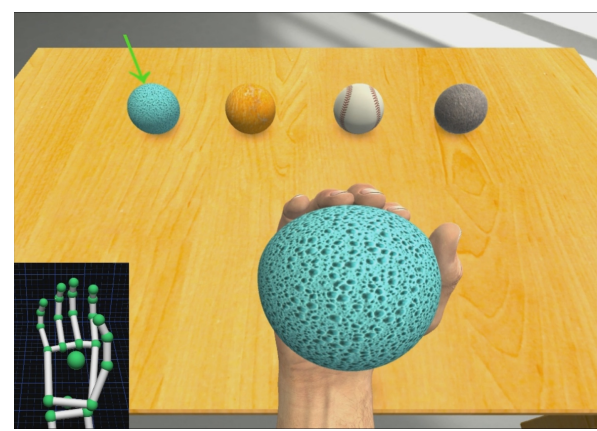

(a)

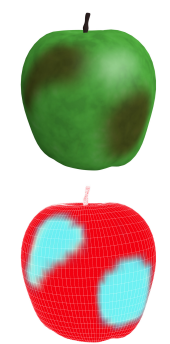

(b)
Figure 4: (a) Virtual environment with deformable objects enhanced via pseudo-haptics. (b) visual and pseudo-haptic models of a virtual apple (darker areas are rotten spots), the mesh data is input to our pseudo-haptic algorithm.

done in computer graphics for colors or normal vectors. In practice, the $s_{n}$ coefficient associated to finger $n$ thus varies depending on the position of the finger over the object, which yields varying sensations. For instance, Figure $4 \mathrm{~b}$ shows a virtual fruit with rotten spots and the associated stiffness map that makes those parts softer to the touch.

Figure 4a shows a test environment featuring several deformable balls with various levels of stiffness painted on them depending on their materials. The balls were setup with the following coefficients: 1) foam ball with base stiffness, 2) a fruit $67 \%$ stiffer, 3) a baseball ball $233 \%$ stiffer, and 4) a completely rigid metal (petanque) ball. Equipped with the FlexiFingers device, users can then perceive the varying stiffness of those objects.

\section{Illustrative use cases}

This section presents two use cases that illustrate how our method can enhance 3D interaction and simulate virtual objects with different levels of stiffness in two contexts: medical training and musical learning.

Learning musical instruments - Music learning has been shown to improve when students are exposed to haptic cues, such as vibrations on the wrists and ankles for learning drums [11] and vibrations on the fingers to play piano [12]. In our scenario, we explore how our passive approach can contribute to learning trumpet. A virtual trumpet which valves can be pressed to produce notes is presented to users. For each note of a song, valves are given a weak or a strong stiffness depending on the contribution of the valve to the node (Figure 5a). In this way, valves that are not part of the notes require more effort to push on, and thus it is more difficult to play them wrongly. The influence of this assistance can then be progressively diminished as the training advances, until the player can perform the notes without errors nor assistance. Additional visual feedback can also be enabled to further guide the note learning.

Training in medical palpation - Haptic feedback has a wide range of applications in the medical field, especially when dealing with procedures such as palpation, which goal is to detect anomalies in the shape or consistency of some body part. For instance, existing simulators let trainees explore a virtual body through a desktop haptic interface [27]. Pseudo-haptics has also been used in a similar context by altering the motion of a cursor to suggest relief on the patient's anatomy [6]. Here, we present how our multi-finger pseudo-haptic approach can contribute to this kind of examination. In our scenario, the user can explore the surface of a virtual body, in order to detect an invisible, but stiffer, component (Figure 5b). 


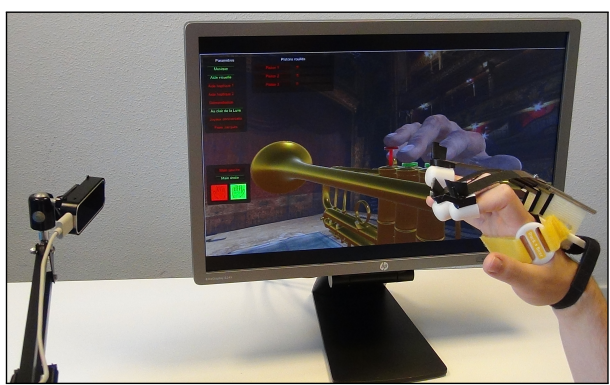

(a)

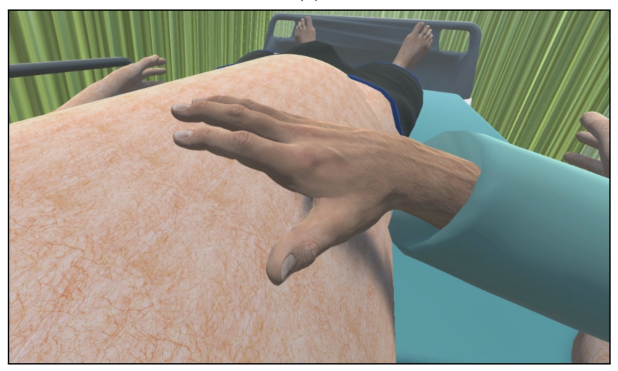

(b)

Figure 5: Illustrative use cases. (a) Trumpet learning: wrong valves (displayed in red on monitor) require more pressing effort thanks to our pseudo-haptic feedback approach (Leap Motion device is visible on the left). (b) Medical palpation training: when exploring a virtual body the user must detect a "hidden" bloated part which is stiffer.

\section{Conclusion}

In this paper, we explored how passive haptics and pseudo-haptic feedback could be associated to support multi-finger interaction. We presented the FlexiFingers, a novel elastic exoskeleton glove that constrains the separate fingers so that each of them can independently move and feel varying forces when manipulating virtual objects. Thanks to its passive nature, this system is a simple and low-cost alternative to the complex haptic devices designed for dexterous interaction. We then proposed an accompanying pseudohaptic approach that leverages visual feedback to modulate the sensations provided by the device. This perception-based technique has already been extensively studied and validated in the literature in similar contexts $[16,2]$. In this way, soft and firm objects can be distinguished and virtual objects with heterogeneous stiffness can be simulated. Several practical use cases related to musical learning and medical training illustrated how this approach could be incorporated into virtual reality scenarios.

Future work could first concern the design of our exoskeleton device. For example, the finger modules could be adjusted to better match the resting posture of the human hand which is slightly curled. Second, we could study how to make use of a tracking system directly embedded on the finger modules, e.g., mechanical bend sensors. In addition, we could also envisage to combine our system with tactile rendering devices $[17,5,22]$. Then, the FlexiFingers approach should be evaluated through a user study in order to validate the stiffness perception. Finally, concrete applications of our approach could be further explored, such as mobile training applications or video games, which often necessitate simple and low-cost input/output devices.

\section{ACKNOWLEDGMENT}

This work was supported by ANR (MANDARIN project, ANR-12CORD-0011).

\section{RefEREnCES}

[1] M. Achibet, A. Girard, M. Marchal, and A. Lécuyer. Elastic-arm: Human-scale passive haptic feedback for augmenting interaction and perception in virtual environments. In Proc. of VR, 2015.

[2] M. Achibet, M. Marchal, F. Argelaguet, and A. Lécuyer. The virtual mitten: A novel interaction paradigm for visuo-haptic manipulation of objects using grip force. In Proc. of 3DUI, pages 59-66, 2014.

[3] Y. Ban, T. Kajinami, T. Narumi, T. Tanikawa, and M. Hirose. Modifying an identified curved surface shape using pseudo-haptic effect. In Proc. of HAPTICS, pages 211-216, 2012.

[4] F. Barbagli, R. Devengenzo, and K. Salisbury. Enabling multifinger, multihand virtualized grasping. In Proc. ICRA, pages 806-815, 2003.

[5] H. Benko, C. Holz, M. Sinclair, and E. Ofek. Normaltouch and texturetouch: High-fidelity $3 \mathrm{~d}$ haptic shape rendering on handheld virtual reality controllers. In Proc. of UIST, pages 717-728, 2016.

[6] L. Bibin, A. Lécuyer, J. Burkhardt, A. Delbos, and M. Bonnet. SAILOR: a 3-d medical simulator of loco-regional anaesthesia based on desktop virtual reality and pseudo-haptic feedback. In Proc. of VRST, pages 97-100, 2008.

[7] C. W. Borst and R. A. Volz. Evaluation of a haptic mixed reality system for interactions with a virtual control panel. Presence, 14(6):677696, 2005.

[8] M. Bouzit, G. V. Popescu, G. C. Burdea, and R. F. Boian. The Rutgers Master II-ND force feedback glove. In Proc. of HAPTICS, pages 145$152,2002$.

[9] G. Casiez, P. Plénacoste, C. Chaillou, and B. Semail. The DigiHaptic, a new three degrees of freedom multi-finger haptic device. In Proc. of $V R$, pages 35-39, 2003.

[10] L. Dominjon, A. Lécuyer, J. Burkhardt, P. Richard, and S. Richir. Influence of control/display ratio on the perception of mass of manipulated objects in virtual environments. In Proc. of VR, pages 19-25, 2005.

[11] S. Holland, A. J. Bouwer, M. Dalgelish, and T. M. Hurtig. Feeling the beat where it counts: fostering multi-limb rhythm skills with the haptic drum kit. In Proc. of TEI, pages 21-28, 2010.

[12] K. Huang, T. Starner, E. Y. Do, G. Weiberg, D. Kohlsdorf, C. Ahlrichs, and R. Leibrandt. Mobile music touch: mobile tactile stimulation for passive learning. In Proc. of CHI, pages 791-800, 2010.

[13] T. Kimura and T. Nojima. Pseudo-haptic feedback on softness induced by grasping motion. In Proc. of EuroHaptics, pages 202-205, 2012.

[14] T. Koyama, I. Yamano, K. Takemura, and T. Maeno. Multi-fingered exoskeleton haptic device using passive force feedback for dexterous teleoperation. In Proc. of IROS, pages 2905-2910, 2002.

[15] P. G. Kry, A. Pihuit, A. Bernhardt, and M.-P. Cani. Handnavigator: hands-on interaction for desktop virtual reality. In Proc. of VRST, pages 53-60, 2008.

[16] A. Lécuyer, S. Coquillart, A. Kheddar, P. Richard, and P. Coiffet. Pseudo-haptic feedback: Can isometric input devices simulate force feedback? In Proc. of VR, pages 83-90, 2000.

[17] J. Martinez, A. Garcia, M. Oliver, J. Molina, and P. Gonzalez. Identifying virtual $3 \mathrm{~d}$ geometric shapes with a vibrotactile glove. IEEE CGA, 36(1):42-51, 2016.

[18] M. Monroy, M. Oyarzábal, M. Ferre, A. Campos, and J. Barrio. MasterFinger: Multi-finger haptic interface for collaborative environments. In Proc. of EuroHaptics, pages 411-419, 2008.

[19] S. Nakagawara, H. Kajimoto, N. Kawakami, S. Tachi, and I. Kawabuchi. An encounter-type multi-fingered master hand using circuitous joints. In Proc. of ICRA, pages 2667-2672, 2005.

[20] C. Pacchierotti, F. Chinello, M. Malvezzi, L. Meli, and D. Prattichizzo. Two finger grasping simulation with cutaneous and kinesthetic force feedback. In Proc. of EuroHaptics, pages 373-382, 2012.

[21] A. Paljic and S. Coquillard. A passive stringed haptic system for immersive environments. In Proc. of EuroHaptics, pages 82-87, 2004.

[22] A. Perez and et al. Optimization-based wearable tactile rendering. IEEE Trans. on Haptics, 2017.

[23] A. Pihuit, P. G. Kry, and M. Cani. Hands on virtual clay. In Proc. of SMI, pages 267-268, 2008.

[24] H. Rijpkema and M. Girard. Computer animation of knowledge-based human grasping. In Proc. of SIGGRAPH, pages 339-348, 1991. 
[25] J. K. Salisbury and M. A. Srinivasan. Phantom-based haptic interaction with virtual objects. IEEE CGA, 17(5):6-10, 1997.

[26] J. Sheng, R. Balakrishnan, and K. Singh. An interface for virtual 3D sculpting via physical proxy. In Proc. of GRAPHITE, pages 213-220, 2006.

[27] S. Ullrich and T. Kuhlen. Haptic palpation for medical simulation in virtual environments. TVCG, 18(4):617-625, 2012.

[28] S. Walairacht, K. Yamada, Y. Koike, and M. Sato. Modeling virtual hands with haptic interface device. Proc. of ICAT, 1(2):1-2, 1999. 